\title{
El desarrollo organizacional como herramienta de apoyo para la organización de bibliotecas digitales: estudio de caso de la Biblioteca del Instituto de Investigaciones en Matemáticas Aplicadas y en Sistemas de la Universidad Nacional Autónoma de México
}

\section{Suyin Ortega Cuevas}

Universidad Nacional Autónoma de México

\section{Resumen}

El presente trabajo busca identificar la manera en que se manifiesta el desarrollo organizacional dentro de las bibliotecas digitales, con el fin de mejorar el proceso y el servicio. Para esto se toman en cuentan las actitudes de los usuarios y las variables que intervienen en este proceso. Entre las primeras están la resistencia al cambio, la motivación y la acertividad, tanto de los usuarios como del personal que participa. En las segundas están la aceptación de los formatos electrónicos y la disponibilidad de los usuarios para su utilización y explotación, y la manera en que son asimilados en el empleo cotidiano por parte de los usuarios para la realización de su actividad académica. Para esta investigación se analiza el Área de Servicios Especializados de la Biblioteca del Instituto de Investigaciones en Matemáticas Aplicadas y en Sistemas (IIMAS). La metodología utilizada es de tipo descriptivo porque en el desarrollo del trabajo se narra la forma en que se manifiestan los cambios en la estructura organizacional en la biblioteca digital, así como el modo en que repercuten en los usuarios, ya sean alumnos o investigadores. Además, se busca especificar los puntos importantes del desarrollo organizacional evaluando las dimensiones del problema expuesto y cuestionado: ¿Los usuarios se adaptan fácilmente a los cambios? ¿Se cumplen las necesidades de información mediante la utilización de la biblioteca digital? ¿La biblioteca digital facilita la obtención de información de manera ágil y efectiva? En el supuesto de que las respuestas sean afirmativas, las preguntas son estas: ¿Los bibliotecarios y usuarios aceptan los cambios de estructuras? ¿Qué los motiva o qué los detiene?

Palabras clave: Desarrollo organizacional. Bibliotecas digitales. 


\section{Abstract}

This paper intends to identify how organizacional development within digital libraries is done, with the purpose of improving the process and the service. The user's attitudes and the variables working on this process are analyzed. The main user's attitudes are resitance to change and strong motivation. The key process factors are the acceptance of the electronic formats, the availability of the system, and the way in which users employ them for the accomplishment of his academic activity. The research was carried out in the Area of Specialized Services of the Library of the Instituto de Investigaciones en Matemáticas Aplicadas y en Sistemas (IIMAS). In addition, some important points of organizacional development are evaluated: Is the user adapted easily to the changes? Are the necessities of information fulfilled by means of the use of the digital library? Digital library facilitates the obtaining of information. Supposing the answers are affirmative the questions are these: Do librarians and users accept the changes of structures? What motivates them or what stops them?

Key words: Organization development. Digital libraries.

\section{Introducción}

El desarrollo organizacional (DO), una herramienta administrativa utilizada desde mediados del siglo pasado, es estudiada, analizada y aplicada en la actualidad en organizaciones lucrativas, donde su función ha desempeñado un papel fundamental en la resistencia al cambio. Ahora es el momento de introducir esta técnica en las bibliotecas, específicamente en la Biblioteca del Instituto de Investigaciones en Matemáticas Aplicadas y en Sistemas (Biblioteca IIMAS).

Por definición, el DO es la manera de manejar el cambio organizacional con el mínimo de conflictos; sin embargo, dado que el factor humano tiene miedo al cambio y que la resistencia resalta por sí sola, la modificación de conducta no se lleva a cabo, lo que da como resultado problemas laborales y provoca que no se implemente el nuevo proceso.

En la Biblioteca IIMAS, el Área de Servicios Especializados realiza las actividades relacionadas con la difusión, la monitorización y actividades vinculadas con los recursos de información electrónicos, ocasionando un contacto constante con los investigadores del Instituto para que hagan uso de estos recursos en sus actividades; sin embargo, se ha visto que el uso no tiene lugar al ciento por ciento, e incluso algunos no los conocen básicamente. Con este antecedente se llevan a cabo unas dinámicas directamente relacionadas con el DO para detectar el problema y modificarlo mediante esta herramienta. 


\section{El Instituto de Investigaciones en Matemáticas Aplicadas y en Sistemas (IIMAS)}

El Instituto de Investigaciones en Matemáticas Aplicadas y en Sistemas (IIMAS) se encuentra adscrito a la Coordinación de la Investigación Científica. Este Instituto realiza investigaciones en diversas áreas del conocimiento, todas ellas aplicadas a las matemáticas, como son la estadística, las ciencias sociales o el cálculo, entre otras. A continuación se describen grosso modo sus características principales.

\subsection{Misión}

Garantizar la existencia de grupos de investigadores en las disciplinas de las Matemáticas Aplicadas, la Ciencia e Ingeniería de la Computación y los Sistemas, para que las mantengan actualizadas y las enriquezcan, contribuyendo así al conocimiento universal de las mismas, además de que provean, tanto al Subsistema de la Investigación Científica como al resto de la comunidad universitaria y a la sociedad, de medios para que estos conocimientos queden a su alcance. (UNAM. IIMAS. Información general, 2004)

\subsection{Objetivos}

Realizar investigación científica original en Matemáticas Aplicadas, en Sistemas y en Ciencias e Ingeniería de la Computación.

Participar, activamente, en los Posgrados: Ciencia e Ingeniería de la Computación, Ciencias Matemáticas y Especialización en Estadística Aplicada. Además, colaborar en los Posgrados en Ingeniería y en el de Ciencias de la Tierra, de los cuales forma parte como entidad académica. (Ibídem)

\subsection{Funciones}

Participar activamente en investigación y en la formación de recursos humanos en los niveles de educación superior, a través de la impartición de cursos, tutoría, dirección de tesis, dentro y fuera de la UNAM, así como de la formación directa de investigadores. (Ibídem)

\subsection{Estructura administrativa}

Dependiendo directamente de la misma Dirección, se encuentran a) la Secretaría Académica (que tiene a su cargo los servicios de apoyo la biblioteca y el área de publicaciones), que "coordina las actividades de los departamentos y de los órganos técnicos y administrativos, con el fin de facilitar la organización, planeación y realización de la investigación, docencia y difusión" (Informe de actividades, 2003, p. 13); b) la Secretaría Técnica, que principalmente tiene que ver con el soporte de cómputo de todo el instituto y la cuestión de logística; "tiene la responsabilidad de proporcionar los servicios técnicos de apoyo al personal y a entidades internas del Instituto" (Informe de actividades, 2003, p. 13); c) finalmente, la Secretaría Administrativa, que tiene a su cargo la administración de la

Scire. $13: 2$ (jul.-dic. 2007) 41-57. ISSN 1135-3716. 
contabilidad y el presupuesto, los suministros para el mantenimiento del edificio y de las actividades y el personal administrativo, y proporciona "el apoyo administrativo necesario para que la dependencia pueda llevar a cabo sus actividades académicas de la manera más eficaz y eficiente, a través de la planeación, organización, coordinación y supervisión de los recursos humanos" (Informe de actividades, 2003, p. 12).

\subsection{Los departamentos}

El Instituto, en cuanto a investigación se refiere, se encuentra dividido en dos áreas: por un lado, la de matemáticas aplicadas y sistemas — "Los investigadores miembros del departamento transitan en las diferentes etapas de su trabajo por varias áreas de la matemática y su aplicación, dependiendo de los problemas científicos que se deseen resolver en un momento dado" (Informe de actividades, 2003, p. 12)—, y por otro, la de ciencia e ingeniería de la computación — “el cómputo científico es una herramienta de gran utilidad para la comprensión de las dinámicas complejas que surgen del estudio de las ecuaciones diferenciales" (Informe de actividades, 2003, p. 12)—. De esta manera se logra combinar unas disciplinas a simple vista tan diferentes que, sin embargo, son complementarias entre sí.

En el área de matemáticas aplicadas y sistemas se encuentran inmersos cuatro departamentos: Matemáticas y Mecánica, Métodos Matemáticos y Numéricos, Modelación Matemáticas de Sistemas Sociales y Probabilidad y Estadística.

Por lo que respecta al área de ciencia e ingeniería de la computación, en ella se concentran los departamentos de Ciencias de la Computación e Ingeniería de Sistemas Computacionales y Automatización.

El personal académico, los investigadores, son un total de 58 y se encuentran distribuidos en diferentes departamentos según sus líneas de investigación, tal y como se muestra en la tabla I.

\begin{tabular}{|l|c|}
\hline Matemáticas y Mecánica & 14 \\
\hline Métodos Matemáticos y Numéricos & 7 \\
\hline Modelación Matemática de Sistemas Sociales & 6 \\
\hline Probabilidad y Estadística & 12 \\
\hline Ciencias de Computación & 8 \\
\hline Ingeniería de Sistemas Computacionales y Automatización & 11 \\
\hline Total & 58 \\
\hline
\end{tabular}

Tabla I. Distribución de personal académico por departamento (fuente: Informe de actividades, 2003). 


\subsection{La Biblioteca del Instituto de Investigaciones en Matemáticas Aplicadas y en Sistemas}

La Biblioteca IIMAS tiene como objetivo "satisfacer las necesidades de información del personal académico del Instituto, de los profesores y estudiantes de los posgrados en que participa el IIMAS y en general de la comunidad universitaria, en las áreas del conocimiento que se cultivan en el Instituto" (Informe de actividades, 2003, p. 235), y como toda organización tiene una misión, la cual se enuncia a continuación:

La Biblioteca del Instituto de Investigaciones en Matemáticas Aplicadas y en Sistemas (BIBLIOTECA-IIMAS) debe ser una entidad dinámica y de vanguardia que incida de manera decisiva, oportuna y eficiente en los procesos de generación, transmisión y difusión de conocimientos en el país, a través de sus servicios". (Ibídem)

Está especializada en matemáticas aplicadas y en sistemas, y su comunidad de usuarios tiene alumnos, profesores, investigadores de diversas universidades e institutos de investigación del país; sin embargo, su comunidad interna la integran 58 académicos y 36 técnicos académicos. Asimismo proporciona servicio a alumnos de las especialidades que se imparten en el Instituto y, en cuanto a otros subsistemas, sirve de apoyo a alumnos de las facultades de Ciencias, Ingeniería y todas aquellas que son de áreas afines.

La Biblioteca IIMAS se encuentra dividida en tres áreas y una jefatura, las cuales tienen las siguientes funciones:

Jefatura: coordinar, organizar, dirigir y evaluar los servicios que ofrece la Biblioteca IIMAS $[\ldots]$.

Servicios Técnicos: adquirir, registrar, procesar y controlar los materiales seleccionados y solicitados por el personal académico del IIMAS.

Servicios al Público: facilitar al personal académico del Instituto y a todos los demás usuarios de la Biblioteca IIMAS, el uso de los acervos con que cuenta. Obtener los materiales solicitados por los académicos del Instituto que se ubiquen en otras bibliotecas del país o del extranjero.

Servicios Especializados: difundir servicios y recursos digitales especializados que apoyen las actividades sustantivas del Instituto. Ofrecer a la comunidad académica del IIMAS información actualizada sobre materiales de interés para ellos y susceptibles de incorporarse al acervo de la Biblioteca IIMAS. (Reglamento de la Biblioteca, p. 2)

Esta investigación solo se centrará en las actividades relacionadas exclusivamente con el área de Servicios Especializados, no por ello olvidando las demás, ya que las tres se complementan entre sí para proporcionar el servicio adecuado. Esta área tiene a su cargo las revistas electrónicas especializadas en matemáticas aplicadas y cuenta con 230 accesos a publicaciones periódicas relativas a matemáticas, ciencia e ingeniería de computación, estadística y sistemas sociales. En total, 56 libros electrónicos, 53 recursos en Internet, 329 revistas electrónicas y 21 bases de datos.

Scire. 13 : 2 (jul.-dic. 2007) 41-57. ISSN 1135-3716. 
Los recursos de información electrónicos con los que cuenta esta área son revistas digitales, libros electrónicos, recursos en Internet y toda aquella información que se brinda a través de la Red (para que los usuarios tengan acceso a la información en texto completo es imprescindible tener una cuenta con IP de la UNAM). Todos estos recursos se encuentran concentrados en la página web de la Biblioteca IIMAS y organizados por tipos. Cabe mencionar que se ha de verificar que los accesos se encuentran funcionando con el fin de garantizar que la información esté disponible; en caso contrario, se deberán realizar los cambios correspondientes. Toda esta actividad es transparente para los investigadores.

El servicio de diseminación selectiva de información (DSI) se encarga de enviar mediante correo electrónico reseñas de libros de acuerdo con el perfil de interés de los investigadores, ya se trate de nuevas ediciones, reimpresiones o materiales de años anteriores; asimismo, se les informa sobre nuevas publicaciones periódicas para que consideren su suscripción.

El servicio de alerta es una recopilación de las tablas de contenidos de las revistas electrónicas y en papel que informa sobre los últimos fascículos recibidos en la Biblioteca IIMAS. Estas revistas electrónicas también pueden ser consultadas desde la página web de la Biblioteca, ya que en ella existe un listado ordenado por temas y alfabéticamente para su fácil localización.

\section{Concepto de desarrollo organizacional}

Diversos autores han definido el desarrollo organizacional, y se puede decir todas las definiciones tienen algo en común: se trata de un cambio planeado a través de la participación del personal. Sin embargo, para contar con la definición de un especialista, reproducimos a continuación algunas de ellas.

Blanchard (1970, p. 2) dice que es "un esfuerzo planeado que abarca toda la organización, administrando desde arriba, para aumentar la eficiencia y la salud de las organizaciones, a través de inversiones planeadas en los procesos organizacionales, usando conocimientos de las ciencias del comportamiento".

Bennis $(1969$, p. 2) lo considera como "una respuesta al cambio, una compleja estrategia educacional con la finalidad de cambiar las creencias, actitudes, valores y estructura en las organizaciones, de modo que estas puedan adaptarse mejor a nuevas tecnologías, nuevos mercados y nuevos desafíos, y al aturdido ritmo de los propios cambios". Las variables a analizar son las siguientes: $a$ ) el cambio rápido e inesperado; $b$ ) el aumento de tamaño de las actividades tradicionales de la organización; c) la complejidad de la moderna tecnología, que requiere armonizar actividades y personas de competencias muy diversas y muy especializadas; $d$ ) una amenaza básicamente psicológica que deriva de un cambio en el comportamiento del alto mando (ibídem, p. 22). Y concluye diciendo que el desarrollo organi- 
zacional es una estrategia planeada que va encaminada a satisfacer las exigencias administrativas de la organización haciendo hincapié en el comportamiento humano, haciendo de intermediario entre el agente del cambio y la organización y compartiendo las metas (ibídem, p. 8).

\subsection{El cambio}

Antes de introducir una estrategia que tenga por objetivo un cambio debe realizarse un trabajo previo de sensibilización con el factor humano: "la implementación del cambio significa que es probable que las personas se vean afectadas y que sean necesarios cambios en las conductas de los empleados" (Hodge, 1998, p. 341) por lo que es importante conocer las siguientes cuestiones: $a$ ) qué se va a cambiar; $b$ ) por qué es necesario dar el paso; $c$ ) qué se espera obtener con el cambio; $d$ ) qué beneficio se obtendrá; $e$ ) cuáles son las ventajas, desventajas o problemas; $f$ ) qué cambios de conducta son necesarios en las actividades o tareas programadas.

\subsection{El ciclo del cambio}

Como toda en organización, existen círculos viciosos que se hacen patentes: cuando se quiere introducir un cambio es cuando surge el miedo por parte de los empleados. Con el fin de que esto pueda ser controlado es necesario detectar el punto adecuado para realizar dicho cambio, y en este caso en concreto es importante analizar el ciclo del cambio.

Para Bennis, el ciclo del cambio se representa mediante la necesidad que existe desde dentro de la organización hacia fuera, y viceversa, se suscita la respuesta de un agente exterior de cambio que aplica conocimientos válidos al sistema cliente. Estos pasos conducen a una mejora continua del producto que se ofrece.

Bennis visualiza el cambio desde los agentes externos, las modificaciones que se manifiestan en el contexto de fuera de la empresa, que obligan a la misma a una revolución en su estructura para poder ser más competitiva. Una vez que los cambios externos se han manifestado, la estructura interna tiene que comenzar el ciclo del cambio: $a$ ) programa de inducción de cambios; $b$ ) mejoramiento de la organización; $c$ ) relación cooperativa; $d$ ) aplicación de conocimientos válidos; $e$ ) retroalimentación; $f$ ) difusión del agente de cambio en el contexto exterior.

Otros autores, como Davis, lo consideran casi siempre como algo que requiere las tres etapas siguientes: $a$ ) la descongelación, que significa que las ideas y las prácticas antiguas deben desecharse para que puedan aprenderse las nuevas; con frecuencia, esta etapa de deshacerse de las prácticas antiguas es tan difícil como la del aprendizaje de las nuevas; $b$ ) el cambio es la etapa en la que las nuevas ideas y prácticas se aprenden de tal manera que el empleado piense y trabaje de acuerdo con ellas; c) la recongelación supone que lo que se ha aprendido se integra a la práctica real. Las nuevas prácticas llegan a convertirse en algo que el empleado 
ejecuta y no tanto en algo que conoce. El simple conocimiento de una práctica no es suficiente (Davis, 1981, p. 221).

Sin embargo, cada fase manifiesta diferentes características; por ejemplo, el introducir nuevas actitudes o formas de pensar y el realizar de manera diferente los procesos entran en la etapa de descongelación: es en esta fase donde se da el primer paso del desarrollo organizacional, todos aquellos aspectos que tengan que ver con la introducción al cambio.

En la etapa del cambio es donde se manifiesta de manera homogénea el proceso; el personal ya está enterado de lo que tiene que hacer y lo está asimilando para poder introducirlo en su trabajo, y la motivación y la autoestima están de por medio.

Finalmente, en la etapa de recongelación ya se encuentra asimilado el proceso, ya está en la práctica; aquí es donde la retroalimentación juega un papel importante para poder continuar con el ciclo, ya que no termina, sino que comienza de nuevo.

\subsection{Resistencia al cambio}

Un punto muy importante del desarrollo organizacional es la resistencia al cambio. Como se vio anteriormente, el DO es el cambio planeado a través del factor humano; pues bien, como en todo cambio, existe una alteración, un miedo a lo desconocido. Siempre se presenta una resistencia a implementar nuevas formas de trabajo o modificar ciertas actitudes, y este caso no es la excepción.

En este apartado, donde se analiza la resistencia al cambio, es importante citar lo que Robbins (1994, p. 689) piensa: "Uno de los resultados más documentados de los estudios de la conducta de los individuos es que las organizaciones y sus miembros se resisten al cambio. En cierto sentido esto es positivo. Ofrece un grado de estabilidad y previsibilidad al cambio. Si no hubiera cierta resistencia el comportamiento organizacional adoptaría las características de una casualidad caótica". Esta es una reflexión válida: no siempre la resistencia al cambio será perjudicial para la organización. Siempre debe existir un equilibrio que permita mantener ubicados los roles de cada estructura departamental; sin embargo, como todos los extremos son malos, también hay que tener en cuenta que la estática puede ocasionar algún conflicto con el cambio.

Toda alteración causa un miedo a lo desconocido, y el DO no es la excepción a la regla. Al querer modificar un comportamiento o implementar un nuevo sistema, se presenta una resistencia al cambio por parte del factor humano. Por simple definición la alteración provoca un cambio de actitudes; si estas no son controladas por mecanismos de sensibilización no se puede llevar a cabo el cambio.

La resistencia al cambio se manifiesta de varias maneras: una puede ser la resistencia individual de las características humanas básicas, como son las costum-

Scire. 13 : 2 (jul.-dic. 2007) 41-57. ISSN 1135-3716. 
bres, la seguridad, los factores económicos, el miedo a lo desconocido, el procesamiento selectivo de información, etcétera. Todo individuo muestra cierto rechazo a la hora de involucrarse en nuevos proyectos cuando afectan a estas cuestiones.

Las propias organizaciones también experimentan en sí mismas una resistencia, que está relacionada con la inercia de la estructura, el enfoque limitado por el cambio. La inercia del grupo, la amenaza para la experiencia, para las relaciones establecidas en el poder o para la asignación establecida de recursos..., todos estos factores pueden propiciar cierta intolerancia a la hora de aceptar un cambio; sin embargo, previendo estos inconvenientes se puede realizar una estrategia adecuada para introducir el cambio.

No existe una receta para que el cambio se lleve a cabo de manera efectiva, pero el visualizar la manera en que se implementará, cuáles son las variables, la participación, los acuerdos, la manipulación positiva y la estrategia coadyuvará a que el proceso sea el adecuado.

\begin{tabular}{|l|l|}
\hline Antiguos objetivos & Nuevos objetivos \\
\hline Un solo uso & Usos múltiples \\
\hline Depende de la tecnología & Trasciende la tecnología \\
\hline Mejores herramientas hacen mejores trabajadores & Mejores trabajadores hacen mejores herramientas \\
\hline $\begin{array}{l}\text { Dependemos de las certezas } \\
\text { de los recursos humanos }\end{array}$ & $\begin{array}{l}\text { Estamos seguros que las personas pueden } \\
\text { ser recursivas (aprenden) }\end{array}$ \\
\hline Búsqueda de la mejor manera & Búsqueda de muchas maneras posibles \\
\hline Encontrar usos para nuestros resultados & Encontrar resultados para nuestros usos \\
\hline Cambiar el mundo según nuestras necesidades & Cambiar nosotros según las necesidades del mundo \\
\hline
\end{tabular}

Tabla II. Necesidades antes y después de un cambio (fuente: Gilbreath, 1990).

\section{Los recursos de información electrónicos}

La biblioteca digital recopila la información en medios electrónicos de modo que se proporcionen los servicios de manera remota y los usuarios puedan acceder a ella sin necesidad de tenerse que desplazar. Pero para lograr está biblioteca se debe considerar en primer lugar qué recursos estarán disponibles, cómo y de qué manera se acercan a los usuarios para que puedan explotarlos como es debido. Lo anterior da pie a su descripción según los estudiosos del tema.

\subsection{Definición}

Durante la última década, diversos estudiosos de la materia han tratado de diferenciar o de igualar los distintos tipos de bibliotecas digitales. Algunos dicen que las bibliotecas electrónicas son aquellas que tienen disponibles los documentos tanto

Scire. 13 : 2 (jul.-dic. 2007) 41-57. ISSN 1135-3716. 
en formato electrónico como en papel; las bibliotecas virtuales, aquellas que tienen todo su material únicamente en la Red, sin ningún otro respaldo, y las bibliotecas híbridas son la combinación de las electrónicas con las virtuales. Para evitar confusiones se analizarán algunas definiciones:

García Camarero y García Melero (2001, p. 27) comentan que la biblioteca digital es una "colección organizada de documentos digitales para cuya consulta se precisa de un ordenador, unos programas informáticos y, en algunos casos, de un sistema de telecomunicaciones compuesto por un módem, una línea telefónica, una empresa que facilite el acceso a las redes telemáticas y unos programas de comunicaciones". Esta definición, completa por sí sola, menciona los elementos indispensables: documentos ordenados, uso de una computadora en red y telecomunicaciones; con estos elementos se lleva a cabo el acceso a los sistemas de información.

Otros autores la definen como "una combinación de servicios, arquitectura, una relación de recursos de información, bases de datos, motores de búsqueda de localización y recuperación de información". O bien se entiende como la administración de una colección de información, con servicios asociados, donde la información es puesta a disposición en formatos digitales a través de una red de comunicaciones. Existen muchas definiciones y muchos autores, y cada uno establece sus propios principios para establecer lo que es la biblioteca digital.

Estos conceptos presentan el modo de acceder a la información mediante dígitos (recordemos los 0011 para transferencia de información) a través de un sistema electrónico. Sin embargo, la Digital Library Federation (DLF) entiende las bibliotecas digitales como organizaciones que proporcionan recursos de información con un personal especializado, que selecciona, cataloga, distribuye y preserva la integridad de los recursos.

La biblioteca digital consiste exclusivamente en el almacenamiento, la organización y la recuperación de información por medios electrónicos a través de servicios digitales. Todos los materiales están procesados y se transfieren de un modo digital (sistema binario) a través de redes. Cada material que concentra este tipo de biblioteca es conocido como recurso electrónico de información, ya se trate de revistas digitales, libros electrónicos, documentos electrónicos. Estos se encuentran almacenados en bases de datos; la información puede ser de tipo numérico o alfabético, o una combinación de ambos, y se puede tener o no acceso al texto completo. Esta información está organizada de tal manera que su almacenamiento y recuperación se realicen de forma fácil y ágil por medios electrónicos.

\subsection{Características}

Las características de los recursos de información electrónicos son palpables a simple vista: se consultan a través de un medio electrónico, no es indispensable 
estar en un sitio determinado ni a una hora fija. Sin embargo, para profundizar en el tema se dan a conocer a continuación las características que se consideran más representativas.

\begin{tabular}{|l|l|l|}
\hline Horario de uso & Servicios tradicionales & Servicios electrónicos \\
\hline $\begin{array}{l}\text { Disponibilidad } \\
\text { de la información }\end{array}$ & $\begin{array}{l}\text { Se ajustan a los horarios de servicio } \\
\text { al número de ejemplares restringido } \\
\text { según las políticas de las bibliotecas }\end{array}$ & $\begin{array}{l}\text { Hay un número de licencias; } \\
\text { sin embargo, el acceso es } \\
\text { simultáneo }\end{array}$ \\
\hline Obtención de documentos & $\begin{array}{l}\text { Tiempo largo de espera debido } \\
\text { al transporte }\end{array}$ & $\begin{array}{l}\text { Envío inmediato por la Red; } \\
\text { la información se recibe en } \\
\text { pocos minutos }\end{array}$ \\
\hline Almacenamiento & Restricción de espacio físico & $\begin{array}{l}\text { Servidores más potentes } \\
\text { para un mayor almacenamiento } \\
\text { de información }\end{array}$ \\
\hline Búsqueda de información & $\begin{array}{l}\text { Acceso a un catálogo y posterior } \\
\text { búsqueda en el estante }\end{array}$ & $\begin{array}{l}\text { Ágil localización y rápida } \\
\text { obtención de la información }\end{array}$ \\
\hline Costo & El material se compra una vez & $\begin{array}{l}\text { Costo por acceso mediante } \\
\text { el uso de licencias }\end{array}$ \\
\hline
\end{tabular}

Tabla III. Tabla comparativa de servicios tradicionales y servicios electrónicos de información (fuente: Chowdhury, 2003; Torres Vargas, 2000).

\subsection{Servicios proporcionados}

Toda biblioteca, por definición, tiene a disposición de los usuarios los materiales de información a través de diferentes servicios, como son el préstamo interno, el externo o el interbibliotecario, entre otros. Los recursos de información electrónicos también cuentan con servicios que se ofrecen dentro y fuera del lugar donde se concentran a través de medios electrónicos en formato digital.

Los servicios que se ofrecen son la consulta a revistas electrónicas en resumen y en texto completo, consulta a bases de datos en resumen y con enlace al texto completo, enlaces seleccionados, envío de documentos, catálogos bibliográficos electrónicos, búsqueda y envío de información localizada en red, publicación de revistas electrónicas, búsqueda de texto completo sobre artículos y libros, acceso a bases de datos en línea, apoyo en la clasificación de materiales, traducción de documentos, obtención de documentos en períodos pequeños de tiempo y búsqueda en línea.

La UNAM, a través de la Dirección General de Bibliotecas, cuenta actualmente con acceso a diferentes recursos de información electrónicos, los cuales se han adquirido por medio de compra, arrendamiento, suscripción o bien de manera gratuita.

Los libros electrónicos que ha adquirido la Universidad se encuentran disponibles en texto completo para consulta de los usuarios, pero tienen ciertas restricciones para respetar el derecho de autor: se puede realizar un determinado número

Scire. $13: 2$ (jul.-dic. 2007) 41-57. ISSN 1135-3716. 
de consultas, se permite imprimir un número de páginas limitado, no se puede descargar de la Red todo el documento y los libros se encuentran monitorizados constantemente por los editores; en caso de descubrirse alguna anomalía, se retira el acceso y se notifica el hecho a la DGB.

Las revistas electrónicas son aquellas que a las que la UNAM se suscribe en papel, y adicionalmente gestiona el acceso electrónico a las que ya cuentan con el mismo. Su consulta está restringida a determinados años, es decir, no se puede acceder a todos los números publicados; generalmente el acceso es a partir de 1998, dependiendo del título y del tipo de suscripción.

Las bases de datos con repertorios de referencias bibliográficas de revistas, que pueden estar disponibles o no en formato electrónico; también sirven para saber en qué biblioteca o hemeroteca del país o del mundo pueden ser consultadas físicamente. Cada base de datos proporciona información diferente, según la especialidad respecto a la que se consulte.

La Biblioteca IIMAS tiene sus propios recursos de información electrónicos, algunos adquiridos con recursos del IIMAS. En la tabla IV se indica con cuántos se cuentan actualmente.

\begin{tabular}{|c|c|c|c|c|c|c|}
\hline & $\begin{array}{l}\text { Ciencias de la } \\
\text { Computación }\end{array}$ & Lingüística & Matemáticas & $\begin{array}{l}\text { Probabilidad } \\
\text { y Estadística }\end{array}$ & $\begin{array}{l}\text { Sistemas } \\
\text { Sociales }\end{array}$ & Divulgación \\
\hline Libros electrónicos & 46 & & 6 & 2 & 2 & \\
\hline Revistas electrónicas & 151 & 4 & 119 & 25 & 32 & 5 \\
\hline Recursos en Internet & 6 & & 8 & 4 & 1 & \\
\hline Bases de datos & 8 & & 4 & & 3 & 6 \\
\hline
\end{tabular}

Tabla IV. Número de recursos de información electrónicos disponibles en la Biblioteca IIMAS según las áreas (fuente: UNAM-IIMAS, 2004).

\section{El desarrollo organizacional en la introducción de los recursos de información electrónicos}

Hasta ahora se han analizado por separado las características de los recursos de información electrónicos y el concepto de desarrollo organizacional. Como parte de este estudio de caso se investiga la manera en que aquellos fueron asimilados, su introducción en el Instituto de Investigaciones en Matemáticas Aplicadas y en Sistemas, utilizando como herramienta el desarrollo organizacional.

\subsection{Objetivo general}

El objetivo es aplicar las técnicas del DO para detectar la resistencia a la utilización de los recursos de información electrónicos, y de esta manera emplear el período del cambio para crear la necesidad de su utilización. 


\subsection{Planteamiento del problema}

El planteamiento de un problema es generalmente una pregunta. De entre las diferentes preguntas que pueden surgir respecto a este tema, sería adecuado cuestionarse lo siguiente: ¿Se cumplen las necesidades de información mediante la utilización de los recursos de información electrónicos? ¿Los recursos de información electrónicos facilitan la obtención de información de manera ágil y efectiva?

\subsection{Metodología}

La metodología utilizada es de tipo descriptivo, porque en el desarrollo del trabajo se narra la forma en que los cambios se manifiestan a la hora de introducir nuevos servicios, así como la manera en que repercuten en los usuarios, ya sean alumnos o investigadores.

Para este caso, como técnica de recopilación de información se ha considerado realizar dinámicas organizacionales tales como la sensibilización al cambio y el juego de roles. Este tipo de técnicas se utilizan porque es necesario conocer la opinión de los investigadores, pero desde la perspectiva de usuarios del servicio, para conocer cómo les gustaría que este se les ofreciera.

$\mathrm{Al}$ tratarse de un estudio de caso y una investigación representativa, se optó por analizar una muestra del personal académico tomando como universo a 58 personas. Pretender hablar con toda la población es muy complejo, ya que los investigadores no tienen horarios de entrada ni de salida y todos ellos imparten clases y llevan a cabo diversas actividades, lo que impide lograr una reunión. Por este motivo se optó por realizar el estudio de manera individual.

Había razones a favor de hacerlo de este modo: a) obtener una mayor comunicación con el personal académico; expresaron sus inquietudes sobre los servicios, analizando cada una las afirmaciones, ya que cada vez que contestaban exponían el porqué de la respuesta; $b$ ) manifestaban cómo les ha ayudado cada servicio y cómo desean que sea en el futuro; $c$ ) también se observó el grado de desconocimiento de los servicios, algo que no se había percibido pero que era necesario saber para poder mejorarlos, por lo que el material que se obtuvo fomentará su fortalecimiento; $d$ ) el acercamiento fue mayor, de modo que se ganó confianza por ambas partes, la del académico y la del prestador del servicio.

Pero también había argumentos en contra: $a$ ) no se pudo obtener una homogeneidad al recopilar la información; $b$ ) fue un tanto cansado y repetitivo explicar las instrucciones a cada uno de los participantes, y en ocasiones no se logró llevar a cabo el procedimiento en su totalidad; $d$ ) los participantes no tuvieron la oportunidad de retroalimentarse entre ellos, y por lo tanto las opiniones no pudieron enriquecerse mutuamente; $e$ ) se observó la resistencia y la incomodidad de algunos de ellos; $f$ ) se puede considerar que los procedimientos quedaron incompletos, aunque un punto importante fue la convivencia entre colegas.

Scire. 13 : 2 (jul.-dic. 2007) 41-57. ISSN 1135-3716. 
El tamaño de la muestra se calculó sin establecer ninguna fórmula, porque la población es muy pequeña; sin embargo, se consideró que si se conseguía entrevistar como mínimo al 20\% de la población sería suficientemente representativo, porque $a$ ) entre colegas se comunican e intercambian puntos de vista sobre los servicios que se proporcionan; $b$ ) los procedimientos se aplicaron, si no a todos, sí a la mayoría de los jefes de departamento o bien a los representantes del comité de la Biblioteca; $c$ ) era más que imposible lograr que todo el personal académico pudiera participar en el proceso; $d$ ) analizando la información se puede observar que las inquietudes son muy similares.

La selección se realizó de manera aleatoria: se contaba con un listado y, siguiendo una tabla aleatoria de números, se iban seleccionando para que todos tuvieran la misma posibilidad de participar; posteriormente se solicitaba una cita a los que habían podido ser localizados, disponían de tiempo y estaban dispuestos a colaborar, y solamente a aquellos que contestaron se les aplicó la técnica de recopilación de información, por lo que ellos mismos se fueron excluyendo.

Para este proceso del cambio se utilizaron como herramientas los dos procedimientos motivacionales que se describen a continuación.

\subsubsection{Sensibilización al cambio}

Para lograr un cambio en una organización se debe comenzar por la sensibilización, que puede llevarse a cabo a través de diversos medios. Uno de ellos son los valores como la ética, la puntualidad, el respecto, la integridad o la honradez, sin olvidar la responsabilidad y la comunicación, entre otros. Para este caso se tomarán como premisas los dos últimos mencionados.

A través de la responsabilidad y la comunicación se puede obtener logros significativos: se transmite a los investigadores el sentido de la responsabilidad al utilizar los recursos de información electrónicos, asimilándolos y explotándolos al máximo, y siempre haciendo una retroalimentación de lo que no entienden o no les satisface, y ellos deben expresar sus comentarios. De esta manera obtienen una respuesta y aceptan mejor estos recursos.

\subsubsection{Juego de roles}

Mediante esta dinámica se pretende invertir los papeles del investigador y el bibliotecólogo, para que ambos puedan ver de qué manera solucionarán las desventajas de los servicios electrónicos de información. Se pretende obtener una visión crítica constructiva y de esta manera lograr una adaptación al cambio.

El juego de roles es un tanto difícil de aplicar, ya que estos procedimientos se llevan a cabo de manera personalizada y no existe una comunicación más amplia debido a la falta de unión entre los participantes. 
En esta dinámica, el punto central está en que el participante no sienta que se deja en sus manos la responsabilidad de “¿y tú qué harías?”. En cierto sentido, es mejor no decirlo directamente, sino un tanto disfrazado: cuáles son sus opiniones y qué consideran conveniente para cambiar o modificar una determinada situación, o qué opinan sobre lo que está ocurriendo. Entonces ellos son parte de la solución.

\subsection{Análisis de los resultados}

En cuanto a los resultados obtenidos con respecto al personal académico, se pueden enumerar los siguientes: $a$ ) no conocen a fondo los servicios, ya que en ese caso hubieran podido enumerar no solo cinco, sino hasta diez ventajas y desventajas de cada servicio electrónico de información; $b$ ) se necesita trabajar mucho más con los investigadores a fin de que conozcan mejor los servicios; si los supieran explotar adecuadamente podrían obtener muchos beneficios para ellos mismos y, en consecuencia, para los alumnos a los que transmiten sus conocimientos; $d$ ) una minoría, cuando no sabían qué contestar, mostraron cierto recelo respecto a los servicios electrónicos de información, y simplemente comentaban que nos los consultaban muy a menudo; pero al aplicar los procedimientos cambiaban de actitud a la hora de señalar los valores de responsabilidad y comunicación, y entonces estaban dispuestos a cooperar: el hecho de poder manifestar sus preocupaciones y decir abiertamente lo que pensaban sobre estos servicios les hacía cooperar más.

La mayor parte del personal académico ha asimilado los cambios sin mayores problemas, identifica fácilmente las ventajas de la utilización de los servicios electrónicos de información y está en situación de conocerlos y explotarlos lo mejor posible.

\section{Conclusiones}

El desarrollo organizacional es una herramienta indispensable para motivar a las personas hacia el cambio, y más aún hacia el cambio tecnológico. Los recursos de información se han modificado debido a la transición del formato en papel al electrónico.

La resistencia al cambio tecnológico ha sido indiscutible y, aunque hubo investigadores que participaron con entusiasmo, fue evidente el rechazo de algunos a utilizar los recursos electrónicos, en tanto que otros mostraron un poco de miedo a decir que no conocían los servicios. Sin embargo, debido a la aplicación de los procedimientos mencionados en los apartados anteriores, se lograron resultados muy positivos.

Como resultado de lo anterior se detectan tres retos: $a$ ) propiciar que la plantilla académica dedicada a la investigación y el personal de apoyo hagan uso de los servicios electrónicos de información; $b$ ) incentivar la utilización de los servicios electrónicos de información haciendo evidentes los beneficios que proporcionan

Scire. 13 : 2 (jul.-dic. 2007) 41-57. ISSN 1135-3716. 
a la plantilla académica en términos de tiempo, pertinencia y relevancia, de modo que el personal se sienta motivado para utilizar habitualmente dichos servicios; y c) explotar al máximo con los usuarios las ventajas que brindan los servicios electrónicos, sin excluir los formatos impresos, debido a que estos últimos aún gozan de un peso académico dominante en algunas disciplinas.

Finalmente, se recomienda, en primer lugar, elaborar un programa permanente de sensibilización e introducción al uso de los servicios electrónicos de información dirigido a todo el personal, destacando los beneficios para el trabajo de investigación y de docencia. De lo anterior se espera que la resistencia al cambio disminuya considerablemente. En segundo lugar, se sugiere utilizar la perspectiva institucional que brinda el desarrollo organizacional como herramienta que beneficia a la institución académica, aunque haya sido creada en instituciones privadas.

\section{Referencias}

Audirac Camarena, Carlos Augusto, et ál. (1994). ABC del desarrollo organizacional. México: Trillas, 1994.

Bennis, Warren G. (1969). Organization development: its nature, origins, and prospects. Reading, Mass.: Addison-Wesley, 1969.

Blanchard, Kennet (1970). La administración y el comportamiento humano. México: Técnica, 1970.

Calhoun, Karen (2003). Technology, productivity and change in librarian technical services. // Library Collections, Acquisitions \& Technical Services. 27 (2003) 281-289.

Chowdhury, Gobinda; Chowdhury, Sudatta (2003). Introduction to digital libraries. Londres: Facet Publishing, 2003.

Corrall, Sheila (1995). Academic libraries in the information society. // New Library World. 96:1120 (1995) 35-45.

Davis, Keith (1981). El comportamiento humano en el trabajo. México: McGraw-Hill, 1981, 6. ${ }^{\mathrm{a}} \mathrm{ed}$.

García Camarero, Ernesto; García Melero, Luis Ángel (2001). La biblioteca digital. Madrid: Arco/Libros, 2001.

Gilbreath, Robert D. (1990). La estrategia del cambio. México: McGraw-Hill, 1990.

González Cornejo, Aurelio (2000). Desarrollo organizacional: la alternativa para el siglo XXI. México: PAC, 2000.

Guizar Montufar, Rafael (1998). Desarrollo organizacional: principios y aplicaciones. México: McGraw-Hill, 1998.

Hodge, Billy J. (1998). Teoría de la organización: un enfoque estratégico. Madrid: Prentice Hall, 1998.

Informe de actividades 2003 [presentado por] Federico O'Reilly Togno. México: Instituto de Investigaciones en Matemáticas Aplicadas y en Sistemas, 2003.

Lafuente López, Ramiro (1999). Biblioteca digital y orden documental. México: UNAM Centro Universitario de Investigaciones en Bibliotecología, 1999. 27-35.

Scire. 13 : 2 (jul.-dic. 2007) 41-57. ISSN 1135-3716. 
Lara, Gonzalo (2004). Algunos aspectos a considerar en el ámbito de las bibliotecas digitales. // Liber: Revista de Bibliotecología. 3:4 (oct.-dic. 2001). http://www.ambac.org.mx/ publicaciones/liber.html (2004-04-30).

Lugo Hubp, Margarita (2001). Cómo afecta a la profesión bibliotecaria el uso de las nuevas tecnologías. // Liber: Revista de Bibliotecología. 3:4 (oct.-dic. 2001). http://www.ambac.org.mx/publicaciones/liber.html (2004-04-30).

Reglamento de la Biblioteca del Instituto de Investigación en Matemáticas Aplicadas y en Sistemas (2001). México: Instituto de Investigaciones en Matemáticas Aplicadas y en Sistemas, 2001.

Robbins, Stephen P. (1994). Comportamiento organizacional: conceptos, controversias y aplicaciones. México: Prentice Hall, 1994.

Torres Vargas, Georgina Araceli (2000). La biblioteca virtual: ¿qué es y qué promete? México: UNAM - Centro Universitario de Investigaciones Bibliotecológicas, 2000.

Torres Vargas, Georgina Araceli (2001). La biblioteca digital: una revisión conceptual. // Liber: Revista de Bibliotecología. 3:4 (oct.-dic. 2001). http://www.ambac.org.mx/ publicaciones/liber.html (2004-04-30).

UNAM-IIMAS. Información general (2004). http://www.iimas.unam.mx/ (2004-04-18).

Scire. $13: 2$ (jul.-dic. 2007) 41-57. ISSN 1135-3716. 